\title{
A REMARK ON THE ODD SCHLICHT FUNCTIONS
}

BY M. S. ROBERTSON

Let $(S)$ denote the class of analytic functions

$$
f(z)=z+a_{2} z^{2}+\cdots+a_{n} z^{n}+\cdots
$$

regular and univalent or schlicht for $|z|<1$, and $(U)$ the subclass of odd schlicht functions

$$
\phi(z)=\left[f\left(z^{2}\right)\right]^{1 / 2}=z+b_{3} z^{3}+b_{5} z^{5}+\cdots .
$$

If $\phi(z)$ is real on the real axis, it has been shown* by J. Dieudonné that for all $n$

$$
\left|b_{2 n-1}\right|+\left|b_{2 n+1}\right| \leqq 2, \quad\left|b_{3}\right| \leqq 1 .
$$

This is not known to be true in the case where the coefficients are complex except for $n=1$. For complex coefficients it is known $\dagger$ that

$$
\left|b_{3}\right| \leqq 1, \quad\left|b_{5}\right| \leqq e^{-2 / 3}+\frac{1}{2} \quad(>1),
$$

from which we could conclude only that

$$
\left|b_{3}\right|+\left|b_{5}\right| \leqq \frac{3}{2}+e^{-2 / 3} \quad(>2) .
$$

It is the purpose of this paper to establish the ineyuality (3) for $n=2$ for the case when the coefficients are complex numbers; and to show further that

$$
\begin{array}{r}
\frac{\left|b_{3}\right|+\left|b_{5}\right|}{2} \leqq\left(\frac{\left|b_{3}\right|^{2}+\left|b_{5}\right|^{2}}{2}\right)^{1 / 2} \leqq 1, \\
\left|a_{3}\right| \leqq 1+\left|b_{3}\right|^{2}+\left|b_{5}\right|^{2} \leqq 3 .
\end{array}
$$

* See J. Dieudonné, Annales de l'Ecole Normale Su.périeure, vol. 48 (1931), p. 318 .

$\dagger$ See M. Fekete and G. Szegö, Journal of the J ondon Mathematical Society, vol. 8 (1933), pp. 85-89. 
The inequality $\left|a_{3}\right| \leqq 3$ is well known,* but the second half of the inequality (6) is new, as far as the author is aware.

Since

$$
a_{n}=\sum_{k=1}^{n} b_{2 k-1} b_{2(n-k)+1}, \quad b_{1}=1,
$$

we have by Schwarz's inequality

$$
\left|a_{n}\right| \leqq \sum_{k=1}^{n}\left|b_{2 k-1}\right|^{2},
$$

and in particular,

$$
\left|a_{3}\right| \leqq 1+\left|b_{3}\right|^{2}+\left|b_{5}\right|^{2} .
$$

It is known $\dagger$ that there exists an absolute constant $A$ such that $\left|b_{2 n+1}\right| \leqq A$ for all $n$. The conjecture of Paley and Littlewood that $A=1$ was found to be false by the example of Fekete and Szegö, who demonstrated the existence of an odd function univalent for $|z|<1$ for which $\left|b_{5}\right|=e^{-2 / 3}+1 / 2>1$. We wish to point out that a weaker statement of the conjecture that $\left|b_{2 n+1}\right| \leqq 1$ might conceivably be true, namely, that

$$
\sum_{k=1}^{n}\left|b_{2 k-1}\right|^{2} \leqq n, \quad b_{1}=1 .
$$

If this weaker conjecture is correct, then by (7) the well known conjecture of L. Bieberbach, $\left|a_{n}\right| \leqq n$, would also be true. To substantiate the weaker conjecture (8), we shall demonstrate here that (8) is true for $n=3$. It is already known to be true for $n=1,2$ by (4).

The method used is that of Fekete and Szegö, $\ddagger$ who employ the representation of the coefficients of (1) obtained by K. Löwner.§ Denoting a continuous function of absolute value unity by $k(t)$, we have the following representation which Löwner obtained for the coefficients:

* See K. Löwner, Mathematische Annalen, vol. 89 (1923), pp. 103-121.

$\dagger$ See R. Paley and J. Littlewood, Journal of the London Mathematical Society, vol. 7 (1932), pp. 167-169.

¥ See M. Fekete and G. Szegö, loc. cit.

\$ See K. Löwner, loc. cit. 


$$
\begin{aligned}
& a_{2}=-2 \int_{0}^{\infty} k(t) e^{-t} d t \\
& a_{3}=4\left[\int_{0}^{\infty} k(t) e^{-t} d t\right]^{2}-2 \int_{0}^{\infty} k^{2}(t) e^{-2 t} d t \\
& b_{3}=\frac{a_{2}}{2}=-\int_{0}^{\infty} k(t) e^{-t} d t, \\
& b_{5}=\frac{a_{3}}{2}-\frac{a_{2}^{2}}{8}=\frac{3}{2}\left[\int_{0}^{\infty} k(t) e^{-t} d t\right]^{2}-\int_{0}^{\infty} k^{2}(t) e^{-2 t} d t .
\end{aligned}
$$

Let

$b_{5}=\left|b_{5}\right| e^{2 i \beta} \quad(\beta$ real $), b_{3}=\left|b_{3}\right| e^{i(\alpha+\beta)} \quad(\alpha$ real $), \quad k(t) e^{-i \beta}=e^{i \theta(t)}$,

where $\theta(t)$ is real and continuous save for a finite number of points. Then

$$
\begin{aligned}
\left|b_{5}\right|= & R\left\{\left[\frac{3}{2} \int_{0}^{\infty} e^{-t} \cdot e^{i \theta(t)} d t\right]^{2}-\int_{0}^{\infty} e^{-2 t} \cdot e^{2 i \theta(t)} d t\right\} \\
= & \frac{3}{2}\left[\int_{0}^{\infty} e^{-t} \cos \theta(t) d t\right]^{2}-\frac{3}{2}\left[\int_{0}^{\infty} e^{-t} \sin \theta(t) d t\right]^{2} \\
& -2 \int_{0}^{\infty} e^{-2 t} \cos ^{2} \theta(t) d t+\frac{1}{2} \\
\left|b_{3}\right|^{2}= & {\left[\int_{0}^{\infty} e^{-t} \cos \{\theta(t)-\alpha\} d t\right]^{2} } \\
& +\left[\int_{0}^{\infty} e^{-t} \sin \{\theta(t)-\alpha\} d t\right]^{2}
\end{aligned}
$$

Since the left-hand side of this equation is independent of $\alpha$, we have

$$
\left|b_{3}\right|^{2}=\left[\int_{0}^{\infty} e^{-t} \cos \theta(t) d t\right]^{2}+\left[\int_{0}^{\infty} e^{-t} \sin \theta(t) d t\right]^{2}
$$

Let $x$ denote the non-negative real root of the equation

$$
\left(x+\frac{1}{2}\right) e^{-2 x}=\int_{0}^{\infty} e^{-2 t} \cos ^{2} \theta(t) d t .
$$


Then, by the theorem of Valiron-Landau, ${ }^{*}$ we have

$$
\left|\int_{0}^{\infty} e^{-t} \cos \theta(t) d t\right| \leqq(x+1) e^{-x}
$$

Let

$$
A=A(x) \equiv\left|\int_{0}^{\infty} e^{-t} \sin \theta(t) d t\right| \leqq 1 .
$$

It follows that $\left|b_{3}\right|^{2}+\left|b_{5}\right|^{2} \leqq P(x)$, where

$$
\begin{aligned}
P(x) \equiv & {\left[\frac{3}{2}(x+1)^{2} e^{-2 x}-\frac{3}{2} A^{2}-2\left(x+\frac{1}{2}\right) e^{-2 x}+\frac{1}{2}\right]^{2} } \\
& +\left[(x+1)^{2} e^{-2 x}+A^{2}\right] \\
= & {\left[\left(3 x^{2}+2 x+1\right) \frac{e^{-2 x}}{2}+\frac{1}{2}-\frac{3 A^{2}}{2}\right]^{2} } \\
& +(x+1)^{2} e^{-2 x}+A^{2} .
\end{aligned}
$$

Case 1. Suppose

$$
0 \leqq A^{2} \leqq \frac{2}{3}\left[\left(3 x^{2}+2 x+1\right) e^{-2 x}+\frac{1}{3}\right]
$$

Then

$$
P(x) \leqq\left[\left(3 x^{2}+2 x+1\right) \frac{e^{-2 x}}{2}+\frac{1}{2}\right]^{2}+(x+2)^{2} \cdot e^{-2 x} .
$$

The maximum of the right-hand side of this inequality is 2 and occurs for $x=0$. Hence in this case

$$
\left|b_{3}\right|^{2}+\left|b_{5}\right|^{2} \leqq P(x) \leqq 2 .
$$

CAse 2. Suppose

$$
\begin{aligned}
\frac{2}{3}\left[\left(3 x^{2}+2 x+1\right) e^{-2 x}+\frac{1}{3}\right] & \leqq A^{2} \\
& \leqq \frac{1}{3}\left[\left(3 x^{2}+2 x+1\right) e^{-2 x}+1\right] .
\end{aligned}
$$

* See E. Landau, Mathematische Zeitschrift, vol. 30 (1929), pp. 608-634, especially pp. 630-632. 
Since $\left|b_{3}\right|^{2} \leqq 1$, we have, within the range of $A^{2}$ in this case,

$$
\begin{aligned}
\left|b_{3}\right|^{2}+\left|b_{5}\right|^{2} \leqq P(x) & \leqq 1+\left[\left(3 x^{2}+2 x+1\right) \frac{e^{-2 x}}{2}+\frac{1}{2}-\frac{3 A^{2}}{2}\right]^{2} \\
& \leqq 1+\left[\frac{1-3\left(3 x^{2}+2 x+1\right) e^{-2 x}}{6}\right]^{2} \\
& <2, \quad \text { (for all } x \geqq 0) .
\end{aligned}
$$

Case 3. Suppose

$$
\frac{1}{3}\left[\left(3 x^{2}+2 x+1\right) e^{-2 x}+1\right] \leqq A^{2} \leqq 1 .
$$

Then

$$
\begin{aligned}
\left|b_{3}\right|^{2}+\left|b_{5}\right|^{2} \leqq P(x) & \leqq 1+\left[\left(3 x^{2}+2 x+1\right) \frac{e^{-2 x}}{2}+\frac{1}{2}-\frac{3 A^{2}}{2}\right]^{2} \\
& \leqq 1+\left[\left(3 x^{2}+2 x+1\right) \frac{e^{-2 x}}{2}+\frac{1}{2}-\frac{3}{2}\right]^{2} \\
& \leqq 2, \quad \text { (for all } x \geqq 0) .
\end{aligned}
$$

Since these cases exhaust all those possible, we have

$$
\left|b_{3}\right|^{2}+\left|b_{5}\right|^{2} \leqq 2,
$$

and the equality sign occurs for the function $z /\left(1-e^{i \alpha} z^{2}\right)$.

An application of Schwarz's inequality gives also

$$
\left|b_{3}\right|+\left|b_{5}\right| \leqq 2\left(\frac{\left|b_{3}\right|^{2}+\left|b_{5}\right|^{2}}{2}\right)^{1 / 2} \leqq 2 .
$$

The University of Chicago and Yale University 